\title{
Erratum
}

\section{A Straightforward Synthesis of 2-(1-Vinyl-1H-pyrrol-2-yl)-1H-benzimidazoles from 1-Vinyl-1H-pyrrole-2-carbaldehydes and $o$-Phenylenediamine}

Boris A. Trofimov,* Andrei V. Ivanov, Elena V. Skital'tseva, Alexander M. Vasil'tsov, Igor A. Ushakov, Konstantin B. Petrushenko, Al'bina I. Mikhaleva Synthesis 2009, 3603.

The advance online publication (e-First) version of this article contained incorrect received and revised data.

These mistakes have been corrected for both the current online version and the print publication. 